\title{
Efecto de la frecuencia de riego en la calidad del césped americano utilizando sistema de riego por goteo subterráneo controlado vía internet
}

\author{
Effect of irrigation frequency on the quality of St. Augustine turfgrass \\ using subsurface drip irrigation system controlled via internet
}

\author{
Anthony Arango ${ }^{1 *}$, David Ascencios ${ }^{2}$, Karem Meza $^{3}$, Edwin Pino $^{4}$
}

\begin{abstract}
RESUMEN
La frecuencia de riego condiciona la calidad del césped americano (Stenotaphrum secundatum), cuando la humedad del suelo está cercana a la capacidad de campo y el agua está retenida en el suelo con menor energía. Esta condición hídrica del suelo exige riegos frecuentes y fraccionados para la planta, provocando una mayor demanda de la mano de obra y tiempo que es superada con la automatización y telecontrol de bajo costo. El objetivo del trabajo fue evaluar el efecto de la frecuencia de riego para el césped americano utilizando un sistema de riego por goteo subterráneo automatizado y telecontrolado vía internet. La investigación se realizó en la ciudad de Lima, Universidad Nacional Agraria La Molina. En el diseño de la automatización y telecontrol se utilizaron las plataformas de Arduino y Blynk, sensores, actuadores y microcontroladores. El diseño experimental fue completamente al azar con cuatro tratamientos y tres repeticiones: T1 - frecuencia de riego de 2 veces al día; T2 - frecuencia de riego 3 veces al día; T3 - frecuencia de riego 1 vez al día; T4 - frecuencia de riego cada 3 días. El índice de vegetación diferencial normalizado, la distribución de uniformidad de humedad en el suelo, crecimiento de la hoja de césped, contenido de humedad de suelo, cobertura vegetal y peso de hoja seca y húmeda fueron evaluados en el periodo de marzo a junio del 2019. El mejor tratamiento fue el T2 para el césped americano debido a que mantuvo la humedad del suelo cercana a la capacidad de campo: el NDVI obtenido alcanzó valores de 0,65. La automatización y telecontrol del sistema de riego por goteo subterráneo permitió mejorar la gestión del agua, ahorro de tiempo y mano de obra.
\end{abstract}

Palabras clave: frecuencia de riego, césped americano, riego por goteo subterráneo, Arduino y sensores.

\begin{abstract}
The irrigation frequency establishes the quality of St. Augustine turfgrass (Stenotaphrum Secundatum), when soil moisture content is close to field capacity and water is retained in the soil with less energy. This hydric soil condition requires frequent and fractional irrigations, causing a greater demand for labor and time that is overcome with the low cost of automation and remote control. The objective of the work was to evaluate the effect of the irrigation frequency for the St. Augustine turfgrass using an automated and remote-controlled subsurface drip irrigation system via Internet. The research was carried out in the city of Lima, Universidad Nacional Agraria, La Molina. The Arduino and Blynk platforms, sensors, actuators, and microcontrollers were used for the design of automation and remote control. The experimental design was completely randomized, four treatments and three repetitions. The T1 - irrigation frequency of 2 times a day; T2 - irrigation frequency 3 times a day; T3 - irrigation frequency 1 time a day; T4 - irrigation frequency every 3 days. Normalized differential vegetation index, soil moisture distribution uniformity, turfgrass leaf growth, soil moisture content, plant cover, dry and wet leaf weight were evaluated from March to June in 2019. The best treatment was T2 for St. Augustine turfgrass, because it maintained soil moisture close to field capacity, the NDVI obtained values of 0.65. The automation and remote control of the subsurface drip irrigation system made it possible to improve water management, saving time and labor.
\end{abstract}

Keywords: irrigation frequency, St. Augustine turfgrass, subsurface drip irrigation, Arduino and sensors.

\footnotetext{
1 Unidad de Riego Tecnificado, Universidad Nacional Agraria La Molina (UNALM). La Molina. Lima, Perú.

2 Departamento de Recursos Hídricos, Universidad Nacional Agraria La Molina (UNALM). La Molina. Lima, Perú.

3 Recursos Hídricos, Universidad Nacional Agraria La Molina (UNALM). La Molina. Lima, Perú.

4 Departamento de Ingeniería Civil, Universidad Nacional Jorge Basadre Grohmann, Tacna, Perú.

* Autor por correspondencia: aarango@lamolina.edu.pe
}

Fecha de Recepción: 21 de Abril, 2021.

Fecha de Aceptación: 10 de Junio, 2021. 


\section{Introducción}

La ciudad de Lima - Perú tiene un clima árido, con una precipitación anual de $10 \mathrm{~mm} / \mathrm{año}$ y una evapotranspiración potencial de $827 \mathrm{~mm} /$ año (Ascencios et al., 2020). Por esta razón es importante la gestión del agua para riego de las áreas verdes urbanas y agrícolas, aplicando una correcta programación de riego y el uso de las nuevas tecnologías para el monitoreo y control (Risco et al., 2018).

Según Eisenberg et al. (2014), las áreas verdes urbanas existentes en Lima metropolitana tienen un índice de 3,5 $\mathrm{m}^{2} /$ habitante, para una población de 8,69 millones de habitantes. De acuerdo con la Organización Mundial de la Salud (2016), se deben implementar mayores áreas verdes para el beneficio de la población, y esta idea es reforzada por los objetivos de desarrollo sostenible. Sin embargo, el crecimiento urbano desordenado y la mala planificación urbana no han contemplado nuevas áreas verdes, resultando en un hábitat urbano de baja calidad (Berrocal, 2020).

Las áreas verdes son indispensables para un desarrollo urbano sostenible, definen el atractivo de la ciudad y el mejoramiento de la calidad de vida de los habitantes. Actualmente estas zonas son utilizadas en forma intensiva como espacios de esparcimiento para mantener el bienestar de la población ante la depresión y ansiedad por el confinamiento (Honey-Rosés et al., 2020). Además, ofrecen beneficios importantes como la reducción de las islas de calor, regulación de las emisiones de gases de efecto invernadero, generación de espacios para la actividad física e interacción mental (Lee et al., 2015).

Según Ascencios et al. (2019), el césped americano ocupa el $80 \%$ de las áreas verdes urbanas de Lima. En verano el césped requiere $41 / \mathrm{m}^{2}$, y debido a la escasez del recurso hídrico, el césped se desarrolla bajo un estrés controlado $\left(3 \mathrm{l} / \mathrm{m}^{2}\right)$ para mantener una calidad visual adecuada.

La programación de riego con la frecuencia y lámina adecuadas permite el ahorro del recurso hídrico. El riego intermitente permite fraccionar la lámina de agua, para mantener la humedad cercana a capacidad de campo, y no constituye una limitación la capacidad de retención del suelo como los arenosos, pedregosos y sustratos. Además el riego frecuente reduce el gasto de energía que ejerce la planta para obtener el agua del suelo (Ramadan et al., 2013). Sin embargo, el fraccionamiento de la lámina y su aplicación demanda mayor tiempo y mano de obra en la operación del sistema de riego, por lo que es necesaria la automatización y telecontrol.

En la agricultura de precisión, la automatización de los sistemas de riego es una práctica utilizada para el adecuado control y monitoreo de la presión, caudal, humedad, variables climáticas, incremento de la productividad y calidad del cultivo. Además permite reducir los tiempos en la operación, disminuir costos en la mano de obra y obtener la información de parámetros hidráulicos y sensores en tiempo real para su análisis y toma de decisiones (Tamilselvan et al., 2019; Yin y Li, 2017). Los sistemas de riego presurizado utilizan la automatización y las nuevas tecnologías como el internet de las cosas, para controlar, monitorear, programar y visualizar la información almacenada, en cualquier momento y lugar (Martínez et al., 2019). Actualmente existen tecnologías emergentes como las plataformas de libre acceso Arduino y Blynk, que permiten crear prototipos utilizando sensores y actuadores de bajo costo y fácil acceso para los sistemas de automatización y telecontrol (Ascencios et al., 2020; Montoya et al., 2017). El objetivo del trabajo fue evaluar el efecto de cuatro frecuencias de riego en la calidad del césped americano, utilizando un sistema de riego por goteo subterráneo automatizado y telecontrolado vía internet, con las plataformas libres de Arduino y Blynk.

\section{Materiales y métodos}

La investigación se realizó en un área experimental de la Universidad Nacional Agraria La Molina (UNALM) y tuvo dos etapas. En la primera etapa de noviembre 2018 a febrero 2019 se desarrolló la automatización y el telecontrol del sistema de riego por goteo subterráneo, que fue diseñado e implementado con sensores, actuadores, microcontroladores, accesorios y una conectividad vía internet. En la segunda etapa de marzo a junio del 2019 se evaluó el efecto de la frecuencia de riego en la calidad del césped. El experimento tuvo un diseño completamente al azar (DCA), con 4 tratamientos de frecuencia de riego y 3 repeticiones. El tamaño de la unidad experimental fue de 2,25 m x 1,3 m. En el cálculo de la lámina aplicada se utilizó el factor de césped de 0,8 para 
el verano y 0,4 para el otoño, para las condiciones del distrito de La Molina.

Diseño, calibración y programación del prototipo para la automatización y telecontrol vía internet del riego por goteo subterráneo (RGS).

La automatización y el telecontrol del riego por goteo subterráneo se realizaron considerando las actividades en la operación del sistema de riego: el control del nivel del agua en el tanque, el encendido y apagado del sistema de bombeo, el monitoreo de presiones y caudales en el cabezal, el control de las electroválvulas en las unidades experimentales y el monitoreo de la humedad del suelo.

El microcontrolador fue seleccionado tomando en cuenta los siguientes criterios: la compatibilidad con los sensores y actuadores, el medio de comunicación y la cantidad de pines de ingreso y salida. Los sensores y actuadores se escogieron con los criterios utilizados en la investigación de Ascencios et al. (2020). La conectividad fue vía internet a través de un módem que brindó conexión alámbrica e inalámbrica.

El diseño electrónico se desarrolló considerando los requerimientos de voltaje y amperaje de los microcontroladores, módulo Ethernet, sensores y actuadores. En el diseño electromecánico se consideró el calibre del cableado, el diseño del tablero de arranque directo, así como los voltajes y amperajes de las fuentes de alimentación para la electrobomba.

La información de los sensores o actuadores fue obtenida en milivoltios $(\mathrm{mV})$ y miliamperios $(\mathrm{mA})$. El código de programación se escribió en el lenguaje $\mathrm{C}++\mathrm{y}$ las lecturas digitales fueron obtenidas en bits y/o hertz. Se realizó la calibración relacionando las lecturas digitales con los valores físicos propios de la variable de medición como presión, caudal y humedad. Las curvas de calibración fueron insertadas en el código de programación.

El código de programación fue desarrollado para la activación de los sensores y actuadores, que trabajaron de forma simultánea, con los códigos de seguridad y alerta, integrados en un solo "sketch" propio del Arduino.

Los protocolos de comunicación Transmission Control Protocol/ Internet Protocol (TCP/IP) y User Datagram Protocol (UDP) se utilizaron en los códigos de programación para enviar y recibir información en el aplicativo móvil. El aplicativo fue desarrollado con la plataforma libre Blynk.

\section{Implementación y validación del prototipo en el sistema de RGS}

Las consideraciones para la automatización y telecontrol del RGS fueron las siguientes:

- Los módulos relé de las electroválvulas se instalaron a $20 \mathrm{~cm}$ del microcontrolador.

- El sensor de nivel de agua se ubicó en la parte superior del tubo de $160 \mathrm{~mm}$ de diámetro, que fue conectado por vasos comunicantes al tanque de agua.

- Los sensores de presión fueron colocados al final de la red hidráulica, antes y después del filtro de anillos.

- El sensor de caudal se ubicó a la salida del cabezal de riego.

- Los sensores de humedad del suelo fueron instalados a $10 \mathrm{~cm}$ de profundidad en cuatro unidades experimentales.

- El microcontrolador alámbrico fue instalado cerca del router en el cabezal de riego y el microcontrolador inalámbrico se ubicó en las unidades experimentales dentro del radio de alcance de 30 metros del Wi-Fi.

El aplicativo móvil desarrollado se utilizó en las pruebas de conexión y funcionamiento de los sensores y actuadores vía internet, en tiempo real.

\section{Evaluación de las frecuencias de riego}

La programación de riego se formuló sobre la base de la evapotranspiración potencial obtenida de la estación meteorológica automática Climate Minder de la marca RainBird. Las frecuencias y los horarios de riego fueron los siguientes: T1 - frecuencia de riego intermitente de 2 veces al día (7:00 am y 5:00 pm); T2 - frecuencia de riego intermitente de 3 veces al día (7:00 am, 5:00 pm y 11:00 pm); T3 - frecuencia de riego de $1 \mathrm{vez}$ al día (7:00 am); T4 - frecuencia de riego cada 3 días (7:00 am). La lámina diaria fue fraccionada en los tratamientos $\mathrm{T} 1$ y $\mathrm{T} 2$ y acumulada de los tres días en el tratamiento $\mathrm{T} 4$.

El análisis de varianza (ANOVA) fue usado para verificar el nivel de significancia al 5\% con la prueba de Tukey. Se utilizó el software InfoStat versión 2017 desarrollado en la Universidad de Córdova, Argentina. 


\section{Evaluación de las variables del césped}

Las variables evaluadas fueron las siguientes: distribución de uniformidad de la humedad (DU), crecimiento de la hoja de césped, relación de la humedad del suelo y la energía de retención del agua, peso de la producción de biomasa, cobertura verde e índice de vegetación diferencial normalizado (NDVI).

La DU fue calculada sobre la base de la humedad del suelo obtenida con el sensor TDR 350 (Spectrum Technology, Reino Unido), que utiliza el principio de la reflectometría en el dominio del tiempo. Se realizaron 24 mediciones en cada unidad experimental (UE), con una periodicidad de muestreo de 15 días.

El crecimiento de la hoja de césped se midió en centímetros tomando como referencia el nivel del suelo. Se realizaron 6 mediciones en cada UE, con una periodicidad de 15 días o mensualmente.

La humedad del suelo se obtuvo con el sensor GS1 (Decagon Device, U.S.), que utiliza el principio de la reflectancia en el dominio de la frecuencia (FDR). Los datos de humedad del suelo fueron almacenados en el datalogger EM-50 Solar y en el microcontrolador ESP 32 Módulo Dev Kit, que permitió el envío de los datos de humedad del suelo al aplicativo móvil.

Los pesos frescos y secos del césped fueron medidos con una balanza electrónica después de la poda con la finalidad de controlar la producción de biomasa de cada tratamiento. Se realizó la toma de datos cada 15 días.
El porcentaje de cobertura verde se calculó con el software Fiji and Image G versión 2.0 (Schindelin et al., 2012). Para ello se capturaron fotografías utilizando las bandas RGB del espectro electromagnético. Posteriormente el porcentaje de cobertura verde fue valorado entre 1 y 9 , adoptando la metodología de Leinauer et al. (2014).

El NDVI fue cuantificado con el Green Seeker (Trimble, U.S.). La medición se realizó con una periodicidad de 15 días antes de la poda. Se hicieron 09 mediciones en cada unidad experimental, a una altura de un metro de la superficie del césped.

La calidad visual de césped se evaluó con la metodología propuesta por el Programa Nacional de Evaluación de Césped de Estados Unidos (NTEP, por sus siglas en inglés). Esta metodología fue desarrollada por Morris y Shearman, 1998, quienes propusieron el rango de puntuación de la calidad de césped de 1 a 9 . Con el propósito de realizar una calificación objetiva, utilizando las nuevas tecnologías, se hizo un análisis de regresión para evaluar la correlación entre la calidad visual con la metodología del NTEP y el NDVI.

\section{Resultados y discusiones}

\section{Selección de dispositivos y microcontroladores}

La primera etapa de la investigación comprendió el desarrollo de la automatización y telecontrol del RGS. En la Figura 1 se presenta el diagrama de interacción de los componentes.

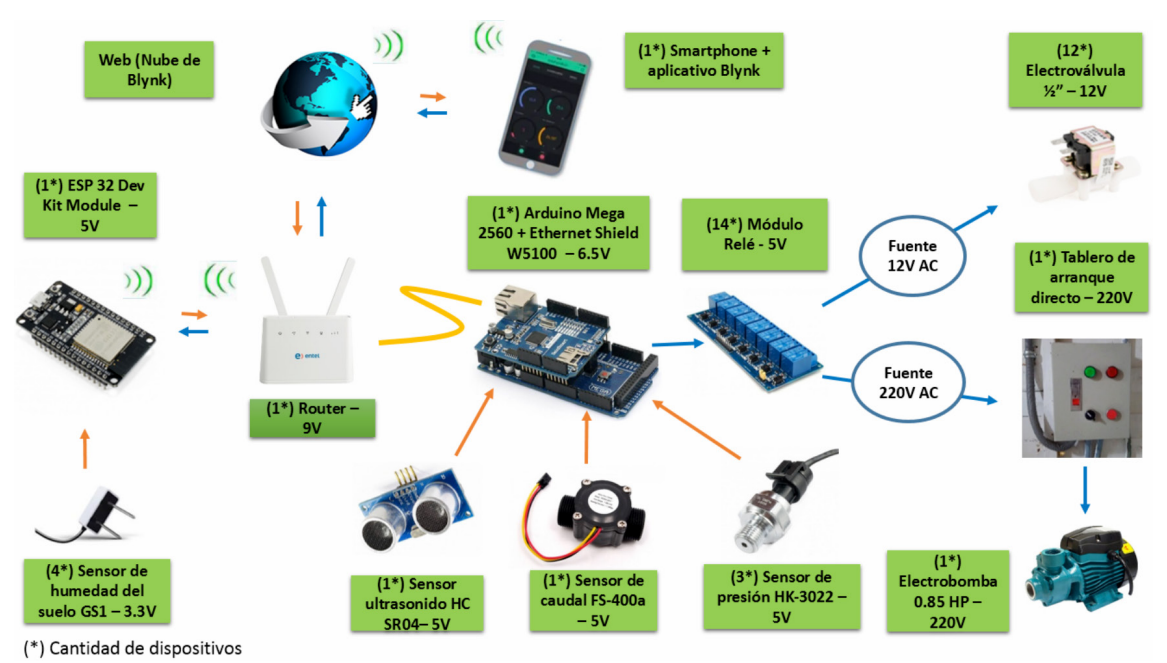

Figura 1. Diagrama de interacción de los componentes del sistema automatizado. 
Los módulos relé de 4 y 8 canales (MS, China) fueron seleccionados para el control de la electrobomba y electroválvulas, y conectados a las fuentes con voltajes de $220 \mathrm{~V}$ y $12 \mathrm{~V}$ respectivamente. La electrobomba consumió 2,54A y las electroválvulas 1A cada una. Según (Mahesh y Raghava, 2016), los módulos relé trabajan con 5 voltios para conectar o desconectar las líneas con voltajes de 12 y 220 voltios, similar a lo ejecutado en la investigación.

El sensor de humedad GS1 (Decagon Device, U.S.) fue seleccionado por presentar una estructura hermética que no permite el paso de la humedad a la parte electrónica, logrando permanecer enterrado por más de un año sin tener desperfectos.

El sensor de caudal (SAIER, China) con diámetro de 1" y rango de medición de 0 a 60 lt/ min permitió monitorear los caudales.

El sensor de presión (KACISE, China) permitió medir las presiones de 0 a 5 bares, con una precisión de $+/-1$ metro de columna de agua.

El sensor de ultrasonido (MANORSHI, China) seleccionado permitió realizar las mediciones de distancias en un rango de trabajo de $2 \mathrm{~cm}$ a $300 \mathrm{~cm}$ con una precisión de $+/-1 \mathrm{~cm}$. El sensor de ultrasonido detecta el nivel de agua en un tanque y cuando llega al mínimo envía señales al módulo relé para apagar la electrobomba y que no trabaje en vacío, evitando el calentamiento.

El Arduino Mega 2560 (Robotlinking Technology, China) fue seleccionado por su compatibilidad con los actuadores y sensores, como se muestra en la Figura 1. El microcontrolador presentó la cantidad suficiente de pines digitales (43) y analógicos (15) para conectar los 15 actuadores y los 8 sensores del sistema de riego. Para enviar la información al aplicativo móvil se utilizó un router de internet que fue conectado de forma alámbrica a una placa adicional que se implementó en el Arduino Mega 2560. Se obtuvo un correcto envío de datos a la nube como lo comprobaron Montoya et al. (2017).

Para las conexiones de los cuatro sensores de humedad de suelo se utilizó el microcontrolador ESP 32 Dev Kit Module (Private owner, China), que tiene la capacidad de detectar la señal Wi-Fi y enviar información del sensor.

\section{Desarrollo de la codificación y calibración de sensores}

El lenguaje de programación del Arduino en el entorno de desarrollo integrado (IDE) fue utilizado para la interacción de los sensores y actuadores con los microcontroladores. Antes de programar se seleccionó cada pin del microcontrolador para un determinado sensor o actuador que irá conectado mediante los cables (jumpers). Aademás se desarrolló la codificación para cada dispositivo. En la Figura 2 se muestra el diagrama de flujo de procesos de interacción entre los sensores y actuadores con los dos microcontroladores, las alarmas y el medio de envío de información al aplicativo móvil. Este diagrama de flujo fue utilizado para el desarrollo de la codificación de los procesos.
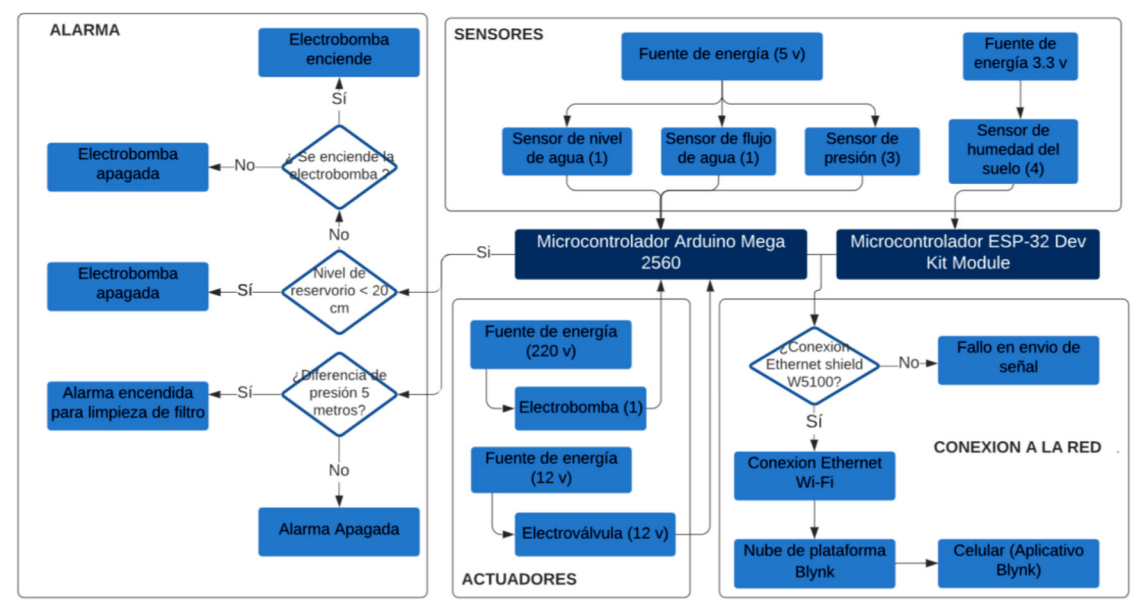

Figura 2. Diagrama de flujo de los procesos de interacción entre los sensores y actuadores con los microcontroladores. 
Se desarrollaron las siguientes alarmas como medida de control y mantenimiento:

- Bloqueo del encendido de la electrobomba cuando el nivel del agua del tanque es menor o igual a $30 \mathrm{~cm}$, a fin de evitar el trabajo en vacío y el recalentamiento de la electrobomba.

- Envío de un mensaje al aplicativo, cuando la diferencia de presión en los transductores antes y después de los filtros es mayor o igual a 5 metros, para la limpieza del filtro de anillas.

Los sensores de presión, nivel del agua, caudal y humedad del suelo fueron calibrados obteniendo ecuaciones lineales con coeficientes de correlación desde 0,86 hasta 0,99 , como se muestra en la Tabla 1.

La calibración del sensor de presión obtuvo un $R 2$ de 0,98 , semejante al valor alcanzado en la investigación de Arasu (2018), que encontró una relación lineal entre las variables y un $R 2$ de 0,99 .

La calibración del sensor de ultrasonido presentó un $R 2$ de 0,99 en el rango de operación de 0,02 a 2 metros. Se utilizó la lectura del sensor para generar ecuaciones y obtener información de los volúmenes de agua en el tanque, en litros. Montoya et al. (2017) utilizaron el sensor de nivel de agua por su confiabilidad y transformaron las unidades de longitud a volúmenes para su mejor comprensión.

La calibración del sensor de caudal presentó un $R 2$ de 0,93 , cercano a lo encontrado por Arasu (2018), que obtuvo un $R 2$ de 0,98 en una regresión lineal relacionando hertz y litros/segundos.

El sensor de humedad del suelo presentó un $R 2$ de 0,86 . La calibración en verano fue más rápida y completa por el amplio rango de humedades en el suelo. Según Peters et al. (2013), la calibración se debe realizar en el lugar donde se instalará el sensor porla variabilidad del suelo.

El desarrollo de la codificación de los sensores, actuadores y microcontroladores, la selección del medio de comunicación y las curvas de calibración, permitió que se implementaran las interfaces y los gráficos para el aplicativo móvil con la plataforma Blynk. En la Figura 3 se muestran las interfaces de los actuadores para el control del encendido y apagado de la bomba y la programación de riego de las electroválvulas.

La plataforma Blynk permitió añadir herramientas, denominadas "widgets", para la visualización gráfica de los valores del sensor en una línea de tiempo y su almacenamiento posibilitó la evaluación posterior del sistema de riego como se observa en la Figura 3. Según Athil et al. (2017), los valores monitoreados del caudal le permiten al usuario identificar los caudales de cada turno de riego y corregir los caudales anormales provocados por una fuga de agua u obstrucción de goteros. De acuerdo con Tamilselvan et al. (2019), con la plataforma Blynk se puede realizar la programación de riego, el monitoreo y visualización de los registros almacenados desde un aplicativo vía internet.

\section{Implementación del sistema}

En el sistema de riego automatizado vía internet se instalaron los siguientes componentes: 12 electroválvulas (una en cada UE), 3 sensores de presión, 1 sensor de caudal, 1 sensor de ultrasonido y 4 sensores de humedad del suelo, como se muestra en la Figura 4.

Los sensores de humedad del suelo GS1 fueron instalados para cada tratamiento y validados con el método gravimétrico. Susha Lekshmi et al. (2014) afirman que los sensores que utilizan el principio de FDR son confiables cuando se realiza una correcta instalación.

El sensor de ultrasonido HC-SR0 se instaló en un tubo de PVC de $160 \mathrm{~mm}$ de diámetro, conectado mediante vaso comunicante con el tanque de 1100 litros.

Tabla 1. Curva de calibración de los sensores.

\begin{tabular}{llc}
\hline Sensor analógico & \multicolumn{1}{c}{ Curva de calibración } & Coeficiente de correlación $\left(\mathrm{R}^{2}\right)$ \\
\hline Presión & $\mathrm{P} 1(\mathrm{bar})=0,0166 * \mathrm{X} 1-2,026$ & 0,98 \\
Nivel de agua & $\mathrm{P} 2(\mathrm{~cm})=0,9799 * \mathrm{X} 1+1,5658$ & 0,99 \\
Caudal & $\mathrm{P} 3(\mathrm{lt} / \mathrm{s})=0,2417 * \mathrm{X} 2+1,318$ & 0,93 \\
Humedad de suelo & $\mathrm{P} 4(\%)=0,0368 * \mathrm{X} 1-44,593$ & 0,86 \\
\hline
\end{tabular}

P1, P2, P3, P4 = Valores físico, X1 = Valor analógico del sensor en bits, X2 = Valor en hertz. 

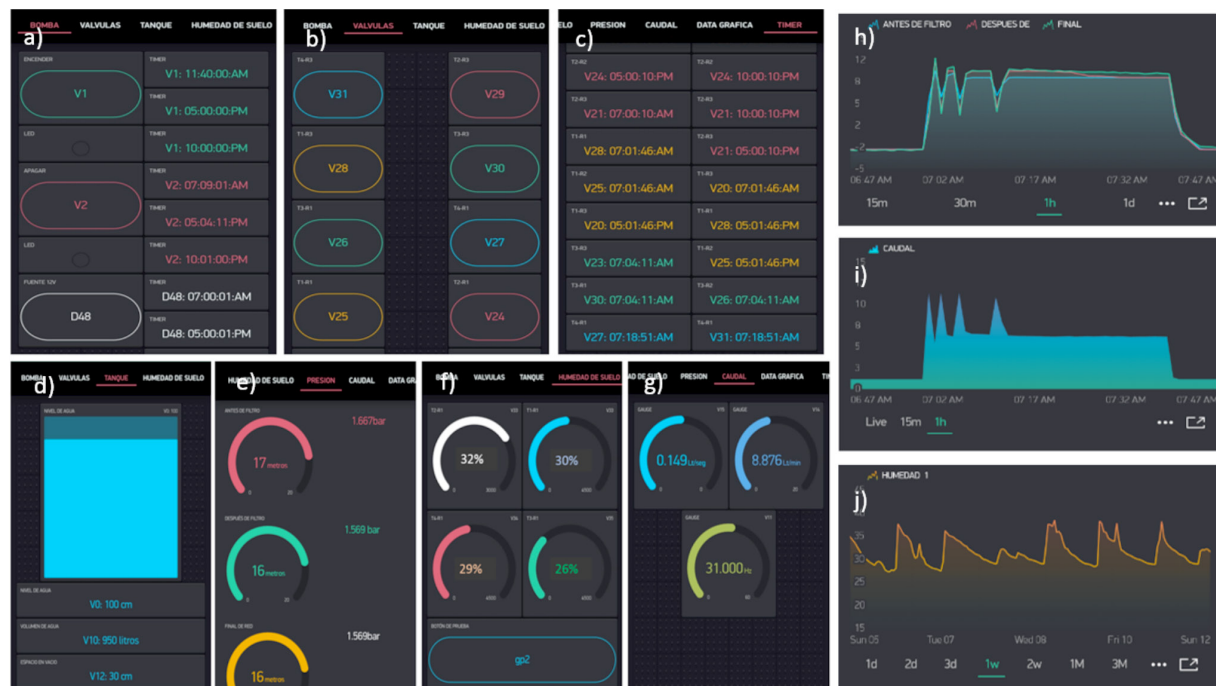

Figura 3. Control de bombeo (a), control de electroválvulas (b), programación de riego (c), nivel de agua (d), presión del sistema (e), humedad de suelo (f), caudal de sistema (g), data histórica de presión (h), data histórica de caudal humedad de suelo (i), data histórica de humedad de suelo (j).
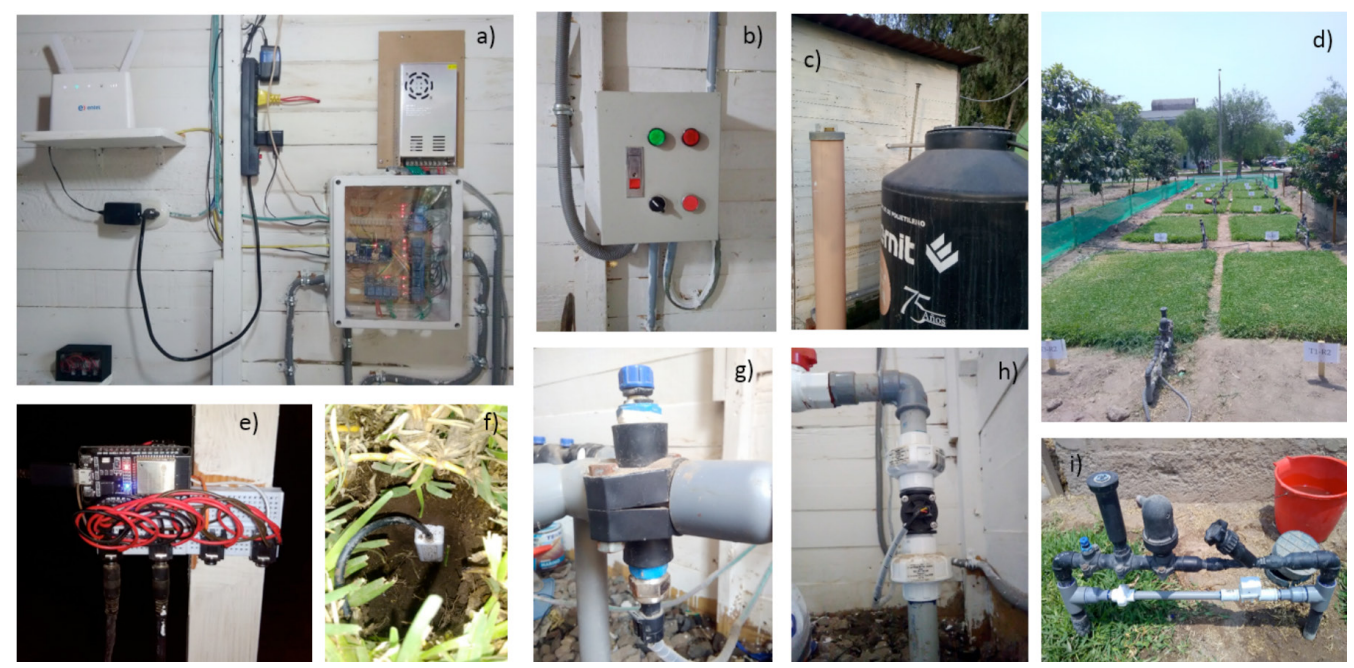

d)

Figura 4. Instalación del prototipo automatizado para el RGS - Programador, fuente de energía y módem de internet (a), tablero de arranque directo (b), sensor de nivel de agua en tubo de $160 \mathrm{~mm}$ (c), unidades experimentales (d), microcontrolador vía Wifi (e), sensor de humedad de suelo GS1 (f), sensor de presión (g), sensor de caudal (h), arco de riego y electroválvula (i)

El sensor de caudal FS-400 a fue instalado después del filtro, en el cabezal de control, y validado con el método volumétrico. Según Athil et al. (2017), el sensor de caudal tiene una alta precisión con un error de $0,39 \%$.

El sensor de presión HK-3022 se validó con un manómetro presentando un error de $1 \%$ a $2,85 \%$. Los sensores expuestos a la intemperie fueron cubiertos con silicona para su protección.
El Arduino Mega 2560 fue instalado en la caseta de riego cercano al módem y el ESP 32 Dev Kit Module en las unidades experimentales dentro del radio de alcance de la señal de Wi-Fi, de 30 metros.

La conectividad del prototipo se probó utilizando el aplicativo instalado en un celular que se conectó a la red 4G. El control y monitoreo del sistema de riego por goteo subterráneo en tiempo 
real se realizó con el aplicativo desde lugares con señal 3G y 4G.

\section{Evaluación de la frecuencia de riego}

La segunda etapa del experimento comprendió la operación del sistema y la evaluación de las cuatro frecuencias de riego. Para determinar la mejor calidad del césped se utilizó la misma lámina de riego para cada tratamiento. La operación del sistema de riego y la evaluación de las frecuencias fueron controladas, monitoreadas y almacenadas mediante el prototipo desarrollado de automatización y telecontrol del RGS. Según Yin y Li (2017), la programación de riego en el césped se mejora implementando un sistema automatizado basado en el internet de las cosas, simplificando el control, reduciendo el uso de la mano de obra y facilitando la gestión del agua de riego.

Los valores de la ETo promedio mensual de los meses marzo, abril, mayo y junio fueron de 2,$9 ; 2,7 ; 1,6$ y $1,2 \mathrm{~mm} /$ día respectivamente. Se presentó un valor máximo diario de $4,4 \mathrm{~mm} /$ día en el mes de abril y el mínimo de $0,7 \mathrm{~mm} /$ día en el mes de mayo. La lámina acumulada de riego que se aplicó en los tratamientos fue de $228 \mathrm{~mm}$, que corresponde a $2,280 \mathrm{~m} 3 / \mathrm{ha}$. Se registró una temperatura máxima de $34{ }^{\circ} \mathrm{C}$ en el mes de marzo y la mínima de $16^{\circ} \mathrm{C}$ en el mes de junio. La precipitación acumulada en los 4 meses fue de $2 \mathrm{~mm}$.

La fuente de agua fue el río Rímac. La conductividad eléctrica fue de $0,73 \mathrm{dS} / \mathrm{m}$, clasificada como C2, es decir, de salinidad moderada. $\mathrm{Su} \mathrm{pH}$ fue de 7,59, clasificado como ligeramente neutro.
El suelo tiene una textura franco arenosa, presenta una capacidad de campo de $28 \%$ y punto de marchitez de $14 \%$ en base del volumen. El pH fue de 9,59, clasificado como ligeramente alcalino. $\mathrm{La}$ conductividad eléctrica promedio fue de 1,50 dS/m, que corresponde a un suelo ligeramente salino. El suelo tiene una porosidad de $47 \%$.

Las variaciones de la humedad del agua en el suelo en el tiempo fueron diferentes para las frecuencias de riego utilizadas como se muestra en la Figura 5. La lámina diaria del $\mathrm{T} 2$ fue fraccionada en tres partes, lo que mantuvo la humedad ligeramente por encima de la capacidad de campo, con una energía de retención del agua en el suelo de $2.5 \mathrm{~m}$, proporcionando una mayor aireación en la zona radicular en comparación con los tratamientos T1 y T3.

El T4 utilizó el 35,71\% de la humedad disponible del suelo y una energía de retención del agua en el suelo de $7 \mathrm{~m}$, alcanzando valores de humedad del $23 \%$. Los T1 y T3 usaron una humedad disponible del $0,5 \%$ y 14,28\%, con energías de retención del agua en el suelo de $1 \mathrm{~m}$ y $4 \mathrm{~m}$ respectivamente. $\mathrm{La}$ aireación del sistema radicular en estos tratamientos fue menor que en el T2, como se muestra en la Figura 5. Cañete-Salinas et al. (2019) mencionan que el riego intermitente mantiene la humedad en capacidad de campo, obteniéndose mejores resultados en el rendimiento, debido a la respuesta fisiológica de la planta.

En la Figura 6 se observa el comportamiento de las variables evaluadas en el césped durante el periodo de marzo a junio de cada tratamiento.

La DU no mostró diferencia significativa entre los tratamientos. El rango de valores fue de $70 \%$ a $94 \%$.

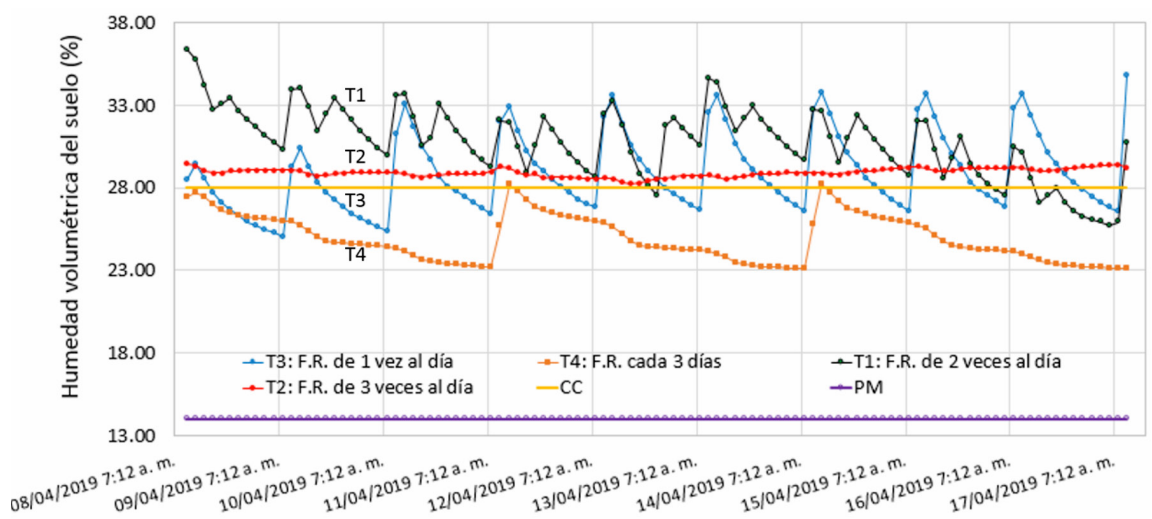

Figura 5. Humedad del suelo para los tratamientos: T1- 2 veces al día, T2- 3 veces al día, T3- una vez al día y T4 cada 3 días. 
El crecimiento de hoja presentó una diferencia significativa con un mínimo valor de crecimiento de $0,2 \mathrm{~cm}$ en el T4, y un valor máximo de $2,6 \mathrm{~cm}$ en el T2 en el mes de marzo, como se muestra en la Figura 6c. En la Figura 6d se observa que el peso fresco obtuvo el valor máximo de $30 \mathrm{gr} / \mathrm{m}^{2}$ en el mes de marzo en el T1 y el menor valor de $4 \mathrm{gr} / \mathrm{m}^{2}$ en el mes de mayo en el T4. Con el T4 se obtuvo el menor costo de mantenimiento debido a la menor frecuencia de riego, menor crecimiento de hoja y menor costo en el transporte del césped. Sin embargo, la calidad visual no fue la adecuada. Adicionalmente, se observa el descenso del crecimiento del césped por el cambio de estación, de verano a otoño. Se comprobó lo mencionado por Jankowski et al. (2018), en cuanto a que hay un mayor crecimiento del césped americano en la época de verano por las altas temperaturas, mientras que en invierno se ve disminuido debido al descenso de la temperatura.

El NDVI presentó diferencia significativa en la 4 ta y 5 ta semana entre tratamientos. Se obtuvo un valor máximo de 0,65 en los meses de abril y junio en el tratamiento T2 y un mínimo valor de 0,44 en el tratamiento T4. Según Pino (2019), si los valores de NDVI están cercanos a 1 , se espera que la planta se encuentre saludable, y para valores cercanos a 0 la planta está estresada o es suelo desnudo. El tratamiento T2 obtuvo una mejor calidad visual en comparación con los otros tratamientos.

La cobertura vegetal no mostró diferencia significativa entre tratamientos. Se obtuvo un valor máximo de $99 \%$ para los tratamientos $\mathrm{T} 1$, T2 y T3 en el mes de marzo y un valor mínimo de $75 \%$ en el tratamiento T4 en el mes de mayo.

La correlación de los valores obtenidos para la calidad del césped utilizando la metodología del NDVI versus la metodología NTEP presentó la siguiente ecuación: NDVI $=0,0294 * \mathrm{NTEP}+0,3684$, con un $R^{2}=0,48$ no significativo. Leinauer et al. (2014) obtuvieron valores de $R^{2}$ entre 0,37 y 0,65 , que indican que la metodología NTEP no puede ser reemplazada con valores de NDVI para determinar la calidad del césped.
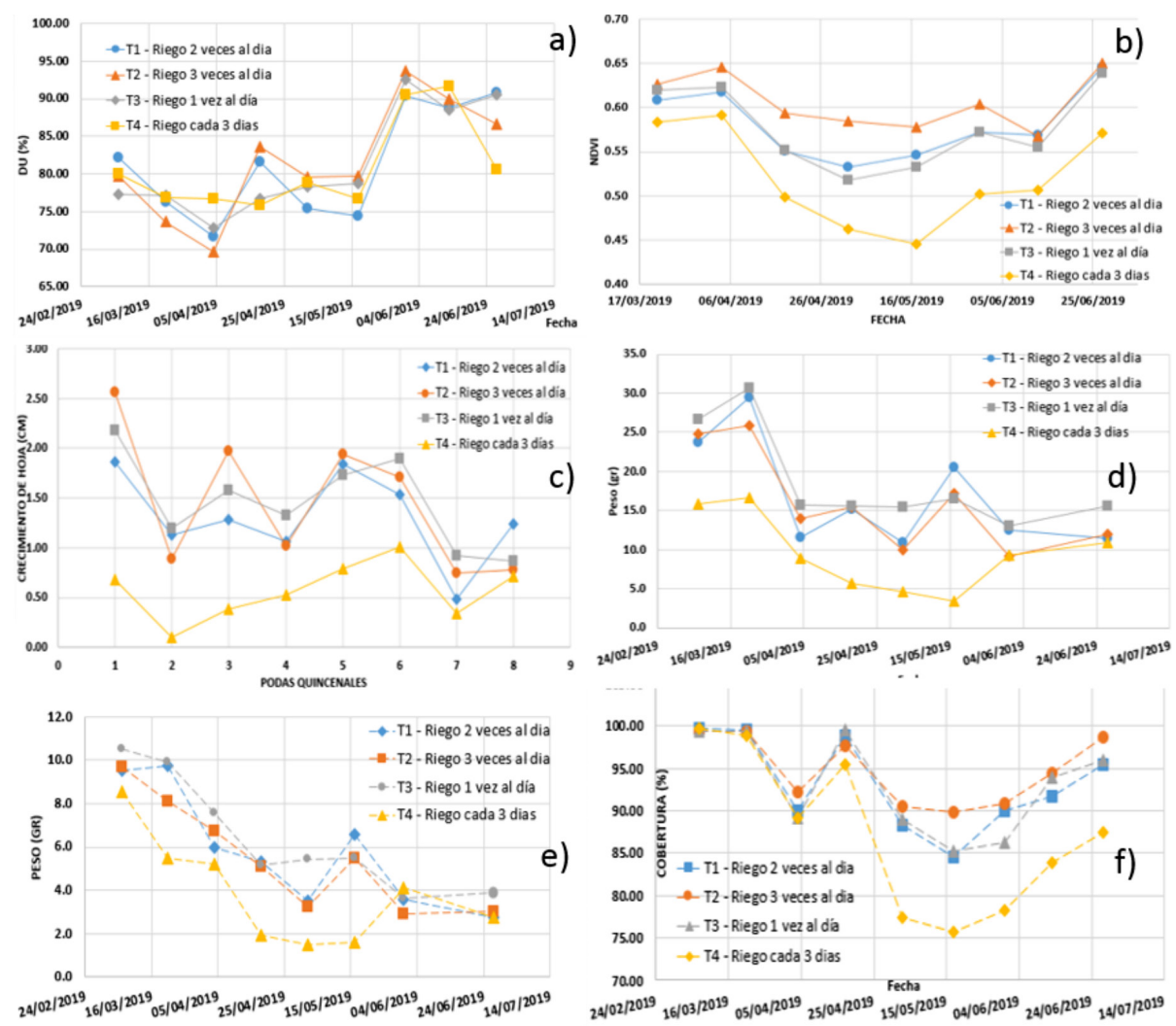

Figura 6. Distribución de la uniformidad de la humedad del suelo (a), índice de vegetación diferencial normalizado (b), crecimiento de la hoja (c), peso de la hoja fresca (d), peso de hoja seca (e), cobertura vegetal (f). 


\section{Conclusión}

Se desarrolló un sistema automatizado y telecontrolado vía internet para el sistema de riego por goteo subterráneo de la UNALM, con la finalidad de facilitar la operación y evaluación del efecto de distintas frecuencias de riego en la calidad del césped. Se seleccionaron e implementaron adecuadamente el Arduino Mega 2560 y ESP 32 Devkit Module V1, los actuadores y los sensores. Se utilizó un aplicativo para el monitoreo y almacenamiento de la presión, caudal, nivel de agua, humedad del suelo, encendido y apagado de electrobomba y electroválvulas, lo que permitió monitorear y detectar fallas del sistema desde cualquier lugar y momento.

El tratamiento T2 (frecuencia de riego: 3 veces al día) fue la mejor opción para el césped americano. Sobre la base de las variables evaluadas se obtuvo un NDVI de 0,65; DU de 85\%. El crecimiento de hoja fue de $2,5 \mathrm{~cm}$ y la humedad de suelo se mantuvo cercana a la capacidad de campo, que brindó una buena aireación y baja energía de retención del agua en el suelo proporcionando la mejor disponibilidad de agua para la planta.

\section{Literatura Citada}

Arasu, K.

2018. Automated experimental procedure using sensors and Arduino. Proceedings of the International Conference on Inventive Computing and Informatics, ICICI 2017. pp. 383-387.

Ascencios, D.; Meza, K., Lluen, J.; Simón, G.

2020. Calibración, validación y automatización del sistema de riego por goteo subterráneo usando un microcontrolador Arduino. Revista de Investigaciones Altoandinas, 22(1): 95-105.

Ascencios, D.; Montalvo, N.; Meza, K.; Cifuentes, A.

2019. Irrigation coefficients of turfgrass (Stenotaphrum secundatum) in arid conditions, under water stress using subsurface drip irrigation. Scientia Agropecuaria, 10(2): 207-216.

Athil, N.M.; Manikandan, S.; Thangamalar, J.B.; Soundaravalli, V. 2017. Monitoring and Analysis of Aquatic Intake in Instructive Bodies. International Research Journal of Engineering and Technology (IRJET), 4(3), 1375-1377.

Berrocal, S.

2020. Resiliencia urbana, crecimiento demográfico y cambio climático: la estructura ecológica y las áreas verdes urbanas vinculadas al río Rímac de Lima. South Sustainability, 1(1), e006 DOI: 10.21142/SS-0101-2020-006

Cañete-Salinas, P.; Zamudio, F.; Yáñez, M.; Gyenge, J.; Valdés,

H.; Espinosa, C.; Acevedo-Opazo, C.

2019. Responses in growth and physiological traits in two Populus $\times$ canadensis clones ('I-214' and 'I-488') submitted to different irrigation frequencies in central Chile. Forest Ecology and Management, 449: 117455. DOI:10.1016/j. foreco.2019.117455

Eisenberg, B.; Nemcova, E.; Poblet, R.; Stokman, A. 2014. Estrategia de Infraestructura Ecológica de Lima (LEIS). Universidad de Stuttgart. Alemania. 321 p.

Honey-Rosés, J.; Anguelovski, I.; Bohigas, J.; Chireh, V.; Daher,

C.; Litt, J.; ... Zapata, O.

2020. The Impact of COVID-19 on Public Space: A Review of the Emerging Questions, 1-20.

Jankowski, K.; Truba, M.; Jankowska, J.; Czeluscinski, W.; Wisniewska-Kadzajan, B.; Malinowska, E.; Matsyura, A.

2018. Effects of soil conditioners on lawn grass growth in different year seasons. Applied Ecology and Environmental Research, 16(4): 3755-3765.
Lee, A.C.K.; Jordan, H.C.; Horsley, J.

2015. Value of urban green spaces in promoting healthy living and wellbeing: Prospects for planning. Risk Management and Healthcare Policy, 8: 131-137.

Leinauer, B.; VanLeeuwen, D. M.; Serena, M.; Schiavon, M.; Sevostianova, E.

2014. Digital image analysis and spectral reflectance to determine turfgrass quality. Agronomy Journal, 106(5): 1787-1794.

Mahesh, A.; Raghava, K.

2016. An android based automatic irrigation system using a WSN and GPRS module. Indian Journal of Science and Technology, 9(30): 4.

Martínez, O.; Arguello, C.; León, J.; Cherres, P.; Cujilema, G. 2019. Prototype of automated irrigation system improves the yield of potatoes (Solanum tuberosum L.) in RiobambaEcuador using wireless network sensors-WSN and 6LoWPAN. Maskay, 9(2): 6.

Montoya, A.P.; Obando, F.A.; Morales, J.G.; Vargas, G.

2017. Automatic aeroponic irrigation system based on Arduino's platform. Journal of Physics: Conference Series, 850: 012003.

Morris, K.; Shearman, R.C.

1998. NTEP Turfgrass Evaluation Guidelines. 5 p.

Peters, T.; Desta, K.; Nelson, L.

2013. Practical Use of Soil Moisture Sensors and Their Data for Irrigation Scheduling. WSU Prosser Irrigated Agriculture Research \& Extension Center. WA, US. 8 p. Pino, E.

2019. Los drones una herramienta para una agricultura eficiente: un futuro de alta tecnología. Idesia, 37(1): 75-84.

Ramadan, A.; Ahmed, B.; Hamed, M.

2013. Effect of pulse drip irrigation and mulching systems on yield, quality traits and irrigation water use efficiency of soybean under sandy soil conditions. Journal Agricultural Sciences, 04(05): 249-261.

Risco, D.; Gutiérrez, A.; Val, J.; León, J., Díaz, A.; Benalcázar,

P.; Prieto, $\mathrm{H}$.

2018. Programación de riego en brócoli (Brassica olerácea L. cv. itálica) en los Andes ecuatorianos. Idesia (Arica): 36(1): 57-63. 
Schindelin, J.; Arganda-Carreras, I.; Frise, E.; Kaynig, V.; Longair, M.; Pietzsch, T.; ... Cardona, A.

2012. Fiji: An open-source platform for biological-image analysis. Nature Methods, 9(7): 676-682.

Susha Lekshmi, S.U.; Singh, D.N.; Shojaei Baghini, M. 2014. A critical review of soil moisture measurement. Measurement: Journal of the International Measurement Confederation, 54: 92-105.

Tamilselvan, G.M.; Ashishkumar, V.; Jothi Prasath, S.; Mohammed Yusuff, S.

2019. IoT based automated water distribution system with water theft control and water purchasing system. International
Journal of Recent Technology and Engineering, 7(4): 151-156.

World Health Organization.

2016. Urban green spaces and health. WHO Regional Office for Europe. Copenhagen, Dinamarca. 80 p.

Yin, Y.; Li, X.

2017. Research of Smart and Remote Sprinkler System for Lawn, 2nd International Conference on Mechatronics and Information Technology. Francis Academic Press, UK. pp. 401-404. 
